\title{
ANTIPHOSPHOLIPIDIC ANTIBODIES IN SARS-COV2 INFECTION: STILL A LOT TO LEARN
}

\author{
Ciprian Jurcuț, Savino Sciascia ${ }^{2}$ \\ "Dr. Carol Davila" Central University Emergency Military Hospital, Bucharest, Romania \\ ${ }^{2}$ Nephrology and Dialysis Universitary Unit, and Center of Research of Immunopathology and \\ Rare Diseases (CMID) San Giovanni Bosco Hospital, and Department of Clinical and \\ Biological Sciences, University of Turin, Turin, Italy \\ Corresponding author: Ciprian Jurcut, \\ E-mail: cjurcut@gmail.com
}

In the current number of the journal, Dima et al. reported a series of three cases of antiphospholipid positivity in the context of SARS-CoV2 infection ${ }^{(1)}$. All the three cases were found to be positive for antiphospholipid antibodies (aPLs), namely anticardiolipin antibodies. The relationship between aPLs and COVID-19 has been largely debated. Critically, the question of whether transient aPLs positivity found in critically ill patients or during times of SARS-CoV2 infection may have a pathogenic role for the development of incident thromboses and lead to antiphospholipid syndrome (APS) has been poorly understood.

Shortly after the beginning of the SARS-CoV2 outbreak, the importance of the thrombotic lesions in the pathogenesis of infection became evident. In this context, a large number of publications speculated a potential role of aPLs in COVID-19 patients. From small series of patients to larger studies, the positivity for different aPLs in COVID-19 was reported $^{(2,3,4)}$.

Among others, Pineton de Cambrun et al reported a high prevalence for aPLs in 25 patients with COVID-19 admitted in intensive care unit. A very high prevalence of lupus anticoagulant (LA) (92\%) was noted in these patients, and a prevalence of $52 \%$ and $12 \%$ was noted for anticardiolipin and anti- $\beta 2$-glycoprotein antibodies, respectively ${ }^{(5)}$. Interestingly, $52 \%$ of patients were double positive and $12 \%$ were triple positive for aPLs. Bertin et al reported a significant high prevalence of IgG aCL antibodies in patients with severe versus moderate COVID-19 disease, with a value above $15 \mathrm{U} / \mathrm{ml}$ being associated with an OR of 8.71 (1.76-73.91, $p=0.017$ ) for severe disease in multivariate analysis ${ }^{(6)}$. Xiao et al reported an overall prevalence of aPLs of $47 \%$ in critically ill patients, with a predominance for $\lg A$ type $(25.8 \%$ for $\operatorname{lgA}$ aCL, $28.8 \%$ for $\lg A$ anti- $\beta 2$-glycoprotein I and $22.7 \%$ for their double positivity $)^{(7)}$. In the same study, the timing of the appearance of aPLs titers was assessed in 10 patients known to be negative at the beginning of the disease. Interestingly, the median time for aPLs appearance was 39 days post disease onset.

In contrast, Galeano-Valle et al reported a low prevalence for anticardiolipin IgM and anti- $\beta 2$ glycoprotein I IgM, estimated around $8.3 \%$ of patients with hospitalized COVID-19 infection and 
venous thromboembolic disease (deep vein thrombosis or pulmonary embolism) ${ }^{(8)}$. A review of the published studies by Gkrouzman et al reported an overall aPLs positivity as high as $58 \%$ in COVID-19 patients, with the majority of the patients being positive for $\mathrm{LA}^{(9)}$. In this analysis, the authors did not find studies evaluating the aPLs at 12 weeks and they underlined that additional clinical information (including ongoing therapy or prior history of positive aPLs/APS before COVID-19) was rarely provided.

From practical points of view, there are some issues needing comments.

First of all, for now, it is important to establish when to test for aPLs in COVID-19 patients. Until now, the extensive screening for all patients is not recommended. Moreover, beyond this large reported range prevalence, it is important to establish the clinical relevance of this positivity and the link with thrombotic events and with general COVID-19 evolution. At this point, we need to emphasize some possible technical caveats important for LA interpretation in the context of COVID-19 infection: the possibility of false positive results in patients treated with anticoagulants; and the fact that some LA techniques interfere with high values of C-reactive protein leading to inaccurate results ${ }^{(10)}$.

Secondly, the question whether the detection of aPLs in the COVID-19 setting should change the clinical management required some considerations. A therapeutic dose of anticoagulants might be needed in the context of clinically overt venous thromboembolic disease. For patients with arterial events, the anticoagulant treatment needs to follow the current therapeutical guidelines. In patients without proven thrombotic events, the choice between antiplatelets agents and anticoagulants need a careful evaluation and must be balanced according to the severity of COVID-19 infection. To date, we do not have solid evidence to support that the detection of aPLs should change the clinical management of patients with COVID-19. Further studies are needed to investigate the clinical benefit of alternative anticoagulation strategies in this scenario. We need to emphasize the fact that, for the moment, until new studies and further guidelines will be published, the use of direct oral anticoagulants is not indicated in the context of high-risk patients with aPLs ${ }^{(11)}$.

Thirdly, the positivity of the aPLs identified in acute disease must should be re-evaluated after 12 weeks, as aPLs during infection can be transitory and of indefinite clinical significance. Consequently, the duration of anticoagulant or antiplatelet treatment should be reassessed accordingly.

Not least, we are still collecting data on the clinical course of COVID-19 in patients previously detected with positive aPLs or APS. It seems reasonable that patients with thrombotic APS on 
anticoagulant treatment should continue ongoing treatment during the COVID-19 infection. In patients with positivity for aPLs but not definite APS, with or without prophylactic antiplatelet therapy, the continuation of this treatment and the initiation of anticoagulant treatment should follow the national COVID-19 antithrombotic guidelines.

Supplementary studies are required in order to address the question whether the detection of aPLs should change the management of patients with COVID-19. To date, evidences are not robust enough to support any additional therapeutic approach. Consequently, testing for aPLs outside research purposes in patients with COVID-19 is not recommended. Nevertheless, data emerging from the current pandemic could represent an opportunity to discover potentially common mechanisms that will provide new insight of both COVID-19 and APS.

\section{References}

1. Dima A, Moroti RV, Popescu DN, Berza I, Parvu DA, Dumitrescu B. Anticardiolipin antibodies in COVID-19 report of three cases. Internal Medicine (Medicina Interna) 2021; in press.

2. Zhang $Y$, Xiao $M$, Zhang $S$, Xia $P$, Cao $W$, Jiang $W$, et al.Coagulopathy and Antiphospholipid Antibodies in Patients with Covid-19. N EnglJ Med 2020;382(17):e38.

3. Bowles L, Platton S, Yartey N, Dave M, Lee K, Hart DP, et al. Lupus Anticoagulant and Abnormal Coagulation Tests in Patients with Covid-19. N Engl J Med2020;383(3):288-290.

4. Le Joncour A, Frere C, Martin-Toutain I, Gougis P, Ghillani-Dalbin P, Maalouf $G$, et al. Antiphospholipid antibodies and thrombotic events in COVID-19 patients hospitalized in medicine ward. Autoimmun Rev 2020;102729.doi: 10.1016/j.autrev.2020.102729.

5. Pineton de Chambrun M, Frere C, Miyara M, Amoura

$Z$, Martin-Toutain l, et al. High frequency of antiphospholipid antibodies in critically ill COVID-19 patients: a link with hypercoagulability? J Intern Med 2020;10.1111/joim.13126.

6. Bertin D, Brodovitch A, Beziane A, Hug S, Bouamri A, Mege JL, et al. Anticardiolipin IgG Autoantibody Level Is an Independent Risk Factor for COVID-19 Severity. Arthritis Rheumatol 2020;72(11):1953-1955.

7. XiaoM, Zhang Y, Zhang S, Qin X, Xia P, Cao W, et al. Antiphospholipid Antibodies in Critically III Patients With COVID-19. Arthritis Rheumatol. 2020;72(12):1998-2004.

8. Galeano-Valle F, Oblitas CM, Ferreiro-Mazón MM, Alonso-Muñoz J, Del Toro-Cervera J, di Natale $M$, et al. Antiphospholipid antibodies are not elevated in patients with severe COVID-19 pneumonia and venous thromboembolism. Thromb Res 2020;192:113-115.

9. Gkrouzman E, Barbhaiya M, Erkan D, Lockshin MD. Reality check on antiphospholipid antibodies in COVID19-associated coagulopathy. Arthritis Rheumatol. 2020 Jul 31.doi: 10.1002/art.41472. Online ahead of print. 10. Schouwers SM, Delanghe JR, Devreese KM. Lupus Anticoagulant ( $L A C$ ) testing in patients with inflammatory status: does C-reactive protein interfere with LAC test results? Thromb Res 2010;125:102104.

11. Tektonidou MG, Andreoli L, Limper M, Amoura Z, Cervera $R$, Costedoat-Chalumeau $N$, et al. EULAR recommendations for the management of antiphospholipid syndrome in adults. Ann Rheum Dis 2019;78:1296-1304. 